\title{
CLINICAL PROFILES AND OUTCOMES OF PATIENTS ADMITTED WITH MODERATE TO CRITICAL CORONAVIRUS DISEASE 2019 (COVID-19)
}

\author{
Sultan Mehmood Kamran, Zill-e-Humayun, Arshad Naseem, Mehmood Hussain, Yousaf Jamal, Waqar Malik*, Salman Saleem \\ Pak Emirates Military Hospital / National University of Medical Sciences (NUMS) Rawalpindi Pakistan, *York Teaching Hospital, Clifton, York, United Kingdom
}

\section{ABSTRACT}

Objective: To evaluate various demographic, clinical, radiological and hematological manifestations of moderate to critical coronavirus disease 2019 (COVID-19) and to assess its complications and outcomes in the Pakistani population.

Study Design: Retrospective observational study.

Place and Duration of Study: Pak Emirates Military Hospital, Rawalpindi, from Apr to Jul 2020.

Methodology: Demographic, clinical, radiological and hematological data of 600 consecutive patients were retrieved and analyzed from hospital registry.

Results: Overall, 449 (74.8\%) patients had at least 1 comorbidity, diabetes mellitus being commonest; 228 (38\%) [95\% CI, 34.1$42 \%$ ]. The most common symptoms were cough; 451 (75.2\%), fever; 450 (75\%) and shortness of breath; 419 (69.8\%). At presentation, $222(37 \%)$ patients had moderate disease, 296 (49.3\%) severe and $82(12.7 \%)$ had critical disease. At admission, 277 $(46.2 \%)$ patients required respiratory support and further $185(30.8 \%)$ patients required treatment escalation later on correla ted with disease severity and age $(p<0.001)$. A total of $92(15.3 \%)$ patients died out of which $38(21.2 \%)$ were on noninvasive ventilation and $36(66.6 \%)$ on invasive ventilation, $(p<0.001)$. Overall survival (OS) was $84.7 \%$; log rank $<0.001$. Mortality was highest in critical disease, 72 (31.3\%) ( $p<0.001)$. A majority of patients, 440 (73.3\%) developed complications, the most common being Cytokine release storm (CRS); $57.5 \%$ respiratory failure; $43.8 \%$ and Acute respiratory distress syndrome (ARDS); $38.8 \%$. Thrombotic events occurred in $106(17.6 \%)$.

Conclusion: Majority of patients with moderate to severe COVID-19 had comorbidities and ended up in various complications.

Keywords: Acute respiratory distress syndrome, Critical disease, Cytokine release storm, Mechanical ventilation, Noninvasive ventilation, SARS-CoV-2.

This is an Open Access article distributed under the terms of the Creative Commons Attribution License (http://creativecommons.org/licenses/by/4.0), which permits unrestricted use, distribution, and reproduction in any medium, provided the original work is properly cited.

\section{INTRODUCTION}

Knowledge of the baseline characteristics and outcomes of coronavirus disease 2019 (COVID-19) is crucial for health and government officials engaged in planning efforts to address local outbreaks. By the end of July 2020, globally >80 million confirmed cases of coronavirus disease 2019 (COVID-19) has been reported of which, 486834 confirmed cases of COVID-19 are recorded in Pakistan ${ }^{1}$. The government of Pakistan responded to this outbreak by implementing smart lockdown and designating some tertiary care hospitals as COVID-19 specific such as Pak Emirates Military Hospital (PEMH). Limited knowledge is available about the baseline characteristics of COVID-19 in Pakistani population compared to population of America $^{2}$ and China $^{3}$ being comprehensively studied. The features and outcome of COVID-19 might be different in Pakistan because there are significant differences between the Pakistani population with that of Americans and Chinese in the context of demographic characteristics

Correspondence: Dr Mehmood Hussain, Department of Medicine, Pak Emirates Military Hospital, Rawalpindi Pakistan

Received: 12 Dec 2020; revised received: 25 Apr 2021; accepted: 26 Apr 2021 and prevalence of non-communicable diseases ${ }^{4}$. For instance, percentage population 65 years and above in America is $16 \%$ compared to $11 \%$ in China and only $4 \%$ in Pakistan according to 2019 estimates from World bank ${ }^{5}$. This study evaluated various clinical, laboratory and radiological manifestations of this deadly infectious disease as well as to see its complications and outcomes in the Pakistani population.

\section{METHODOLOGY}

This retrospective observational study was performed at Pak Emirates Military Hospital (PEMH). The study was approved by Ethics Review Committee via letter no A/28/be/201/2020. In this study, a retrospective analysis of records of all patients admitted with confirmed COVID-19 between April to July 2020 was performed. Non-probability convinience sampling was used. The inclusion criteria included COVID-19 diagnosed by Real Time-Polymerase Chain Reaction (RTPCR) positivity for SARS-CoV-2, and moderate, severe or critical illness in accordance with WHO criteria ${ }^{6}$.

COVID-19 infection was confirmed by RT-PCR sampling results of nasopharyngeal and oropharyngeal swabs at the time of admission. Disease severity 
was defined in accordance with WHO criteria ${ }^{6}$. HRCT chest criteria were defined according to Carotti et al study $^{7}$. ALC $<1 \times 10^{9} / 1$ lymphocytes were considered lymphopenia and thrombocytopenia as platelet $<150 \times$ $10^{9} / 1$. In accordance with Institutional COVID-19 management guidelines, all patients COVID-19 received standard of care (SOC) protocol comprising aspirin, anticoagulation, famotidine, Vit C, Vit D, oral zinc and awake Proning (if $\mathrm{PaO}_{2}<80 \mathrm{mmHg}$ ). All patients with CRS or hypoxemia received either Methylprednisolone $1 \mathrm{mg} / \mathrm{kg}$ or Dexamethasone 6-12 mg/day irrespective of disease severity. Oxygen therapy, noninvasive ventilation (NIV) [Continuous positive pressure airway pressure $8-10 \mathrm{~cm}_{2} \mathrm{O}$ ] and invasive ventilation (IV) were instituted where deemed necessary. Patients were discharged, once they recovered. Recovery was defined by de-escalation of patients from critical and severe to mild, or from moderate to mild, with at least 2 of the following; serum Ferritin $<1000 \mathrm{ug} / \mathrm{ml}$ (and decreasing trend on two consecutive days), LDH normalization, CRP $>50 \%$ - fold reduction (and decreasing trend in two consecutive days), ALC $>1000$. CRS was defined according to National guidelines ${ }^{8}$ for COVID19 given as supplementary table-I.

Respiratory complications, that were recorded, included respiratory failure $\left(\mathrm{PaO}_{2}<60 \mathrm{mmHg}\right)$, Acute respiratory distress syndrome (ARDS) according to Berlin definition'. Post COVID lung was defined operationally by the presence of residual fibrosis in usual interstitial pneumonia (UIP) or fibrotic nonspecific interstitial pneumonia (NSIP) on HRCT chest at the time of discharge with a restrictive pattern on spirometry by American thoracic society/European respiratory society standard criteria. Recorded thrombotic events included pulmonary embolism, Acute Coronary Syndrome (ACS) as (Ischemia on ECG and raised Creatine kinase -MB (CKMB) and/or positive Troponin), Stroke, deep vein thrombosis and acute limb ischemia. Other complications included acute liver injury [ALI] (defined by the rise in ALT $>4$ times ULN), Acute kidney injury [AKI] (urine volume $<0.5 \mathrm{ml} / \mathrm{kg}$ for six hours or rise in creatinine by $>26.5 \mathrm{micromol} / \mathrm{L}$ within $48 \mathrm{~h}$ ). Uncontrolled hyperglycemia was defined as plasma glucose more than 200mg despite use of appropriate doses of insulin and/or oral hypoglycemic drugs for at least $72 \mathrm{~h}$.

According to institutional approach, every COVID-19 case classified into moderate, severe or critical disease either at the time of diagnosis or during admission underwent monitoring and evaluation. Data collection included demographic details, preexisting conditions, symptomatology with their duration, the degree of lung involvement on high-resolution chest $\mathrm{CT}$, cardiac involvement by Electrocardiogram, disease category in accordance with WHO criteria on admission, progression of disease and requirement of respiratory support. Laboratory parameters retrieved included complete blood count (CBC), blood groups, markers of Cytokine release storm (CRS) [Ferritin, lactate dehydrogenase (LDH), C-reactive protein (CRP), Ddimers, Interleukin-6 (IL-6) and absolute lymphocyte count (ALC)] and cardiac biomarkers (qualitative CKMB and Troponin-I). The degree of viral clearance was determined by day 7 and 14 RT-PCR status post admission. Important complications due to SARS-CoV2 and outcome were recorded.

IBM Statistical Package for Social Sciences (SPSS) version 23 was used for statistical analysis. For continuous variables Median and range were used with their differences calculated by Students t-test or MannWhitney U-test. Frequency and percentage were used to express categorical statistics and Chi-square test was used to evaluate differences in categorical variables. Kaplan-Meier test was used for survival analysis and log rank was used to compare difference in the two groups. Cox-proportional hazards were used to generate hazard ratios (HRs) and 95\% confidence intervals (CIs) for the outcome. The $p$-value of $<0.05$ was considered statistically significant.

\section{RESULTS}

A total of 600 patients were included with PCR confirmed COVID-19 having either moderate, severe or critical Disease. The median age of these individuals was 60 years [interquartile range $\{\mathrm{IQR}\}, 51-70]$ (range, 14-91 years [95\% CI, 57.6-59.9]). Overall, 490 (81.7\%) were males [95\% CI, 78.3-84.7\%], similarly distributed across all age groups. A total of 195 (32.5\%) [95\% CI, 28.8-36.4\%] individuals were aged more than 65 years, 300 individuals (50\%) [95\% CI, 21-25\%] aged between 45 and 65 years and 105 individuals (17.5\%) [95\% CI, $14.5-20.8 \%$ ] were younger than 45 years. Overall, 449 (74.8\%) [95\% CI, 71.2-78.3\%] patients had at least 1 comorbidity with most common being Diabetes Mellitus at $228(38 \%)$ [95\% CI, 34.1-42\%]) and Hypertension (HTN) 187 (31.2\%) [95\% CI, 27.5-35\%]. Age wise baseline demographic and clinical features of these individuals are described in table-I.

The median duration of hospitalization was 13 days $\{\mathrm{IQR}\}, 9-15$ days (range, 1-51 days [95\% CI, 11.9813.09] and was significantly correlated with age, $(p=$ 
$0.019)$ and disease severity ( $p=0.03$ ). The most common symptoms at triage were Cough, fever, shortness of breath and Myalgia. Only Shortness of breath was significantly associated with increasing age $(p<0.001)$.
Constitutional symptoms included but not limited to; Fatigue 42 (7\%), diarrhea 41 (6.8\%), insomnia 33 (5.5\%), vomiting $33(5.5 \%)$, anosmia $30(5 \%)$, sore throat 28 $(4.7 \%)$, chest pain $25(4.2 \%)$, headache and altered

Table-I: Demographic and clinical characteristics of patients with COVID-19 in Pakistan.

\begin{tabular}{|c|c|c|c|c|c|}
\hline Age range, years & All & $(0-45)$ & $(45-65)$ & $(>65)$ & $\begin{array}{c}p- \\
\text { value }\end{array}$ \\
\hline No. (\%) & $600(100)$ & $105(17.5)$ & $300(50)$ & $195(32.5)$ & \\
\hline Age (years), Median (range) & $60(12-94)$ & $38(12-45)$ & $57(46-65)$ & $73(66-94)$ & \\
\hline \multicolumn{6}{|l|}{ Gender, n (\%) } \\
\hline Male & $490(81.7)$ & $86(81.9)$ & $249(83)$ & 155(79.5) & \multirow{2}{*}{$<0.001$} \\
\hline Female & $110(18.3)$ & $19(18.1)$ & $51(17)$ & $40(20.5)$ & \\
\hline Contact history, n (\%) & $164(27.3)$ & $28(26.7)$ & $86(28.7)$ & $50(25.6)$ & 0.751 \\
\hline Comorbidity type, $\mathrm{n}(\%)$ & $449(74.8)$ & $40(38)$ & $236(78.6)$ & $173(88.7)$ & $<0.001$ \\
\hline Diabetes Mellitus & $228(38)$ & $15(14.3)$ & $128(42.7)$ & $85(43.6)$ & $<0.001$ \\
\hline Hypertension & $187(31.2)$ & $10(9.5)$ & $94(31.3)$ & $83(42.6)$ & $<0.001$ \\
\hline Ischemic Heart Disease & $115(19.2)$ & $4(3.8)$ & $53(17.7)$ & $58(29.7)$ & $<0.001$ \\
\hline Obstructive Airway Disease & $33(5.5)$ & $2(1.9)$ & $19(6.3)$ & $12(6.2)$ & 0.205 \\
\hline Renal disorders & $25(4.2)$ & $3(2.9)$ & $10(3.3)$ & $12(6.2)$ & 0.234 \\
\hline Malignancy & $11(1.8)$ & $1(0.9)$ & $7(2.3)$ & $3(1.5)$ & 0.618 \\
\hline$>3$ comorbidities & $62(20.3)$ & $10(9.5)$ & $39(13)$ & $11(5.6)$ & $<0.028$ \\
\hline None & $151(25.2)$ & $65(61.9)$ & $64(21.3)$ & $22(11.3)$ & $<0.001$ \\
\hline Symptoms; n (\%) & $600(100)$ & $105(17.5)$ & $300(50)$ & $195(32.5)$ & \\
\hline Fever & $450(75)$ & $69(65.7)$ & $238(79.3)$ & $142(72.8)$ & 0.046 \\
\hline Cough & $451(75.2)$ & $72(68.6)$ & $229(76.3)$ & $150(76.9)$ & 0.224 \\
\hline Shortness of Breath & $419(69.8)$ & $52(49.5)$ & $214(71.3)$ & $153(78.5)$ & $<0.001$ \\
\hline Myalgias & $187(31.6)$ & $28(26.7)$ & $59(19.7)$ & $40(20.5)$ & 0.135 \\
\hline Duration of symptoms in days; median (Inter-Quartile Range) & $7(3.5-11.5)$ & $7(4-10)$ & $7(3.5-10.5)$ & $8(3.5-12.5)$ & 0.686 \\
\hline Duration of fever in days; median (Inter-Quartile Range) & $5(3-7)$ & $5(3.5-6.5)$ & $4(2-6)$ & $5(3-5)$ & 0.604 \\
\hline \multicolumn{6}{|l|}{ Extent of lung involvement on HRCT, n (\%) } \\
\hline Few Ground Glass Opacities & $47(7.8)$ & $15(14.3)$ & $20(6.7)$ & $12(6.2)$ & \\
\hline $10-50 \%$ & $247(41.2)$ & $60(57.1)$ & $115(38.3)$ & $72(36.9)$ & $<0.001$ \\
\hline$>50 \%$ & $306(51)$ & $30(28.6)$ & $165(55)$ & $111(56.9)$ & $<0.001$ \\
\hline \multicolumn{6}{|l|}{ HRCT Pattern; n (\%) } \\
\hline Typical pattern & $502(83.7)$ & $88(83.8)$ & $248(82.7)$ & $166(85.1)$ & \multirow{3}{*}{0.532} \\
\hline Atypical Pattern & $57(9.5)$ & $7(6.7)$ & $31(10.3)$ & $19(9.7)$ & \\
\hline Mixed Pattern & $41(6.8)$ & $10(9.5)$ & $21(7)$ & $10(5.1)$ & \\
\hline Respiratory support at the time of admission, $\mathrm{n}(\%)$ & $277(46.2)$ & $36(34.2)$ & $134(44.6)$ & $110(56.4)$ & \multirow{5}{*}{$<0.001$} \\
\hline None & $323(53.8)$ & $69(65.8)$ & $166(55.3)$ & $85(43.6)$ & \\
\hline Only O2 Support & $194(32.3)$ & $24(22.9)$ & $94(31.3)$ & $76(39)$ & \\
\hline Addition of NIV & $55(9.2)$ & $6(5.7)$ & $25(8.3)$ & $27(13.8)$ & \\
\hline Addition of IV & $28(4.7)$ & $6(5.7)$ & $15(5)$ & $7(3.6)$ & \\
\hline Need for treatment escalation, $\mathrm{n}(\%)$ & $185(30.8)$ & $14(13.3)$ & $95(31.7)$ & $76(39)$ & $<0.001$ \\
\hline Oxygen therapy in liters, Median (Inter-Quartile Range) & $6(0-6)$ & $0(0-6)$ & $6(0-12)$ & $8(2-14)$ & 0.002 \\
\hline Final respiratory support, $\mathrm{n}(\%)$ & $409(68.2)$ & $48(45.7)$ & $212(70.6)$ & $149(76.4)$ & \\
\hline None & $191(31.8)$ & $57(54.3)$ & $88(29.3)$ & $46(23.6)$ & $<0.001$ \\
\hline Oxygen Support & $176(29.3)$ & $33(31.4)$ & $92(30.7)$ & $51(26.2)$ & \\
\hline Non-Invasive Ventilation & $179(29.8)$ & $8(7.6)$ & $89(29.7)$ & $82(42.1)$ & \\
\hline Invasive Ventilation & $54(9)$ & $7(6.7)$ & $31(10.3)$ & $16(8.2)$ & \\
\hline \multicolumn{6}{|l|}{ Initial Disease Category at the time of admission; n (\%) } \\
\hline Moderate & $222(37)$ & $61(58.1)$ & $105(35)$ & $56(28.7)$ & \multirow{3}{*}{$<0.001$} \\
\hline Severe & $296(49.3)$ & $36(34.3)$ & $155(51.7)$ & $105(53.8)$ & \\
\hline Critical & $82(12.7)$ & $8(7.6)$ & $40(13.3)$ & $34(17.4)$ & \\
\hline
\end{tabular}




\begin{tabular}{|c|c|c|c|c|c|c|}
\hline \multicolumn{7}{|c|}{ Final Disease Category; n (\%) } \\
\hline \multirow{3}{*}{\multicolumn{2}{|c|}{$\begin{array}{l}\text { Moderate } \\
\text { Severe } \\
\text { Critical }\end{array}$}} & $188(31.3)$ & $56(53.3)$ & $86(28.75)$ & $46(23.8)$ & \multirow{3}{*}{$<0.00 \mathrm{a}$} \\
\hline & & $182(31.3)$ & $35(33.3)$ & $95(31.7)$ & $52(26.7)$ & \\
\hline & & $230(38.7)$ & $15(14.3)$ & $118(39.3)$ & $97(49.7)$ & \\
\hline \multirow{2}{*}{ PCR Negativity } & Day 7 after admission & $\begin{array}{c}203 / 438 \\
(46.3)\end{array}$ & $\begin{array}{l}23 / 72 \\
(31.9)\end{array}$ & $\begin{array}{c}107 / 214 \\
(50)\end{array}$ & $\begin{array}{c}73 / 152 \\
(48)\end{array}$ & 0.026 \\
\hline & Day 14 after admission & $\begin{array}{c}227 / 302 \\
(75.1)\end{array}$ & $\begin{array}{l}37 / 57 \\
(64.9)\end{array}$ & $\begin{array}{c}112 / 145 \\
(77.2)\end{array}$ & $\begin{array}{c}78 / 100 \\
(78)\end{array}$ & 0.0136 \\
\hline \multicolumn{2}{|c|}{ Duration of Hosp Stay; Median (range) days } & $13(4-51)$ & $11(4-50)$ & $13(6-47)$ & $10(6-51)$ & 0.019 \\
\hline \multicolumn{2}{|l|}{ Mortality, n (\%) } & $92(15.3)$ & $6(5.7)$ & $36(12)$ & $50(25.6)$ & $<0.001$ \\
\hline \multicolumn{2}{|c|}{ Complications, n (\%) } & $440(73.3)$ & $60(57.1)$ & $229(76.3)$ & $151(77.4)$ & $<0.001$ \\
\hline \multicolumn{2}{|c|}{ Cytokine Release Syndrome, n (\%) } & $345(57.5)$ & $51(48.6)$ & $175(58.3)$ & $119(61)$ & 0.105 \\
\hline \multicolumn{2}{|c|}{ Respiratory Failure $\left(\mathrm{PaO}_{2}<60 \mathrm{mmHg}\right) ; \mathrm{n}(\%)$} & $263(43.8)$ & $20(19)$ & 141(47) & $102(61.5)$ & $<0.001$ \\
\hline \multicolumn{2}{|c|}{ Pulmonary Embolism; n (\%) } & $28(4.67)$ & $6(5.8)$ & $13(4.2)$ & $9(4.6)$ & 0.645 \\
\hline \multicolumn{2}{|c|}{ Acute Respiratory Distress Syndrome; n (\%) } & $233(38.8)$ & $16(15.2)$ & $124(41.3)$ & $93(47.7)$ & $<0.001$ \\
\hline \multicolumn{2}{|c|}{ Acute Kidney Injury; n (\%) } & $26(4.3)$ & $5(4.8)$ & $15(5)$ & $6(3.1)$ & 0.574 \\
\hline \multicolumn{2}{|c|}{ Acute Coronary Syndrome; n (\%) } & $59(9.8)$ & $6(5.7)$ & $34(11.3)$ & $19(32.2)$ & 0.250 \\
\hline \multicolumn{2}{|c|}{ Acute liver Injury; n (\%) } & $84(14)$ & $13(12.4)$ & $42(14)$ & $29(14.9)$ & 0.839 \\
\hline \multicolumn{2}{|c|}{ Post COVID lung; n (\%) } & $32(5.3)$ & $4(3.8)$ & $8(2.7)$ & $20(10.3)$ & 0.001 \\
\hline \multicolumn{2}{|c|}{ Septic shock; n (\%) } & $29(4.8)$ & $2(1.9)$ & $18(6)$ & $9(4.6)$ & 0.329 \\
\hline \multicolumn{2}{|c|}{ Hyperglycemia; n (\%) } & $68(10.33)$ & $9(8.6)$ & $39(13)$ & $20(10.3)$ & 0.395 \\
\hline
\end{tabular}

mentation each 21 (3.5\%). The median duration of symptoms in moderate, severe and critical disease, respectively, were 5 vs 8 vs 9.5 days $(p<0.001)$. At triage, 222 (37\%[95\% CI 33.1-41\%]) had moderate disease, 296 (49.3\% [95\% CI 45.3-53.4\%]) severe and 82 (13.7\% [95\% CI 11-16.7\%]) critical disease. Of critical disease, 34 $(41.4 \%)$ of patients were $>65$ years $(p<0.001)$. The number of critical COVID-19 increased to 230 (38.7\%) [95\% CI 34.5-42.4\%]) distributed as 15 (14.3\%), 118 (39.3\%) and $97(49.7 \%)$ among younger to older age groups, respectively, during admission $(p<0.001)$. A total of 277 $(46.2 \%)$ patients required respiratory support at the time of admission significantly correlated with age and disease severity ( $p<0.001$ for both). Further 185 (30.8\%) individuals requiring treatment escalation during hospitalization having a significant association with increasing age and severity, ( $p<0.001$ for each). Among radiological features, typical pattern $502(83.7 \%)$ on HRCT chest and involvement $>50 \% 306$ (51\%) were strongly associated with disease severity, $(p<0.001)$.

A total of $440(73.3 \%)$ patients developed different complication. Among complications, 99 (44.6\%) [95\% CI $37.9-51.4 \%$ ] were in moderate disease category, 260 (87.8\%) [95\% CI 83.6-91.3\%] in severe and 81 (99\%) [95\% CI 93.4-100\%] in critical disease, $(p<0.001)$. The most common complications in this study were CRS, respiratory failure, and ARDS. Less common complications included; ALI, Uncontrolled hyperglycemia, ACS, septic shock, PE, and AKI and all of these were associated with mortality, $(p<0.05)$. Total thrombotic events occurred in 106 (17.6\%) patients (PE; 28, ACS;
59, stroke; 6, DVT; 10, limb ischemia; 3). Although only $59(9.8 \%)$ patients developed ACS, cardiac enzymes were raised in $253(42.1 \%)$ patients. CKMB was high in $141(23.5 \%)$, Troponin-I was positive in $112(18.7 \%)$ patients and ECG showed ischemic changes in 70 (11.7\%). Among ACS, 11 (1.8\%) patients developed ST elevation myocardial infarction (STEMI). Respiratory failure, ARDS and Post COVID lung were significantly associated with increasing age and disease severity, $(p<0.001)$ whereas, complications such as CRS, PE, ACS, AKI, ALI, uncontrolled hyperglycemia, and septic shock were significantly associated with disease severity, $(p<0.001)$ but unrelated to age $(p>0.05)$. Overall, on the basis of reports available, 46.3\% (203/438) patients were PCR negative on day 7 of admission and this viral clearance increased to $75.1 \%(227 / 302)$ on day $14^{\text {th }}$ of admission. Although, PCR positivity on day 7 and 14 was significantly correlated with disease severity $(p<0.001)$ however, it was not associated with mortality $(p$-value $=0.01)$.

A total of $179(29.8 \%)$ patients received NIV and $54(9 \%)$ were on IV. The requirement for NIV or IV at admission was $40(13.3 \%)$ in patients between 45 and 65 years and $34(17.4 \%)$ in patients over 65 years $(p<0.001)$. Later on, during hospitalization requirement of NIV or IV in these age groups climbed up to 120 $(40 \%)$ and $98(50 \%)(p<0.001)$, respectively. A total of 92 $(15.3 \%)$ patients died out of which $38(21.2 \%)$ were on NIV and $36(66.6 \%)$ on IV, $(p<0.001)$. Among non-ventilated patients, $18(4.9 \%)$ died because of complications of disease. Mortality rates increased with increa- 
sing severity of disease; 6 (2.7\%) in moderate, 14 (7.7\%)

$(19.1 \%)$ in females, $(p=0.226)$. The median survival time in severe and $72(31.3 \%)$ in critical disease died, $(p$ in the hospital was 8 days (IQR 1-15 days) (range 1-27

Table-II: Laboratory parameters of 549 patients with COVID-19 admitted with moderate, severe and critical disease in tertiary care hospital of Pakistan.

\begin{tabular}{|c|c|c|c|c|c|}
\hline \multirow{2}{*}{ Characteristics $n=549$} & \multirow{2}{*}{ Median value (range) } & \multicolumn{3}{|c|}{ Disease severity; n (\%) } & \multirow{2}{*}{$p$-value } \\
\hline & & Moderate & Severe & Critical & \\
\hline \multicolumn{6}{|l|}{ Hemoglobin, g/dl } \\
\hline $\begin{array}{l}<13 \\
\geq 13 \\
\end{array}$ & $12.8 \mathrm{~g} / \mathrm{dl}(5.5-17)$ & $\begin{array}{c}67(35.3) \\
121(64.7) \\
\end{array}$ & $\begin{array}{c}74(40.9) \\
107(59.1) \\
\end{array}$ & $\begin{array}{c}97(42) \\
134(58) \\
\end{array}$ & 0.009 \\
\hline \multicolumn{6}{|l|}{ TLC $\times 10^{9} / 1$} \\
\hline $\begin{array}{l}<4 \\
4-10 \\
>10\end{array}$ & $10.8 \times 109 / 1(0.2-68.8)$ & $\begin{array}{c}10(5.3) \\
132(60.6) \\
45(24.1) \\
\end{array}$ & $\begin{array}{c}7(3.9) \\
94(51.9) \\
80(44.2) \\
\end{array}$ & $\begin{array}{c}2(0.9) \\
107(46.3) \\
122(52.8) \\
\end{array}$ & $<0.001$ \\
\hline \multicolumn{6}{|l|}{ Absolute Lymphocyte Count x 109/1 } \\
\hline $\begin{array}{l}<1 \\
>1 \\
\end{array}$ & $900(1116.28 \pm 842.692)$ & $\begin{array}{c}62(34.3) \\
119(65.7) \\
\end{array}$ & $\begin{array}{l}92(58.2) \\
66(41.8) \\
\end{array}$ & $\begin{array}{c}146(69.5) \\
64(30.5) \\
\end{array}$ & $<0.001$ \\
\hline \multicolumn{6}{|l|}{ Neutrophil to Lymphocyte Ratio } \\
\hline $\begin{array}{l}1-3 \\
>3-9 \\
>9 \\
\end{array}$ & $8.3(0.6-58.7)$ & $\begin{array}{l}42(22.5) \\
114(61) \\
31(16.5) \\
\end{array}$ & $\begin{array}{l}32(17.7) \\
77(42.5) \\
72(39.8) \\
\end{array}$ & $\begin{array}{c}13(5.6) \\
110(47.6)) \\
108(46.8) \\
\end{array}$ & $<0.001$ \\
\hline \multicolumn{6}{|l|}{ Platelet $\times 10^{9} / 1$} \\
\hline $\begin{array}{l}<150 \\
150-400 \\
>400\end{array}$ & $209(21-1100)$ & $\begin{array}{c}31(16.6) \\
145(77.5) \\
11(5.9) \\
\end{array}$ & $\begin{array}{l}46(25.4) \\
123(68) \\
12(6.6)\end{array}$ & $\begin{array}{l}80(34.6) \\
141(61) \\
10(4.3) \\
\end{array}$ & 0.001 \\
\hline Raised Cardiac biomarkers, n (\%) & Qualitative & $77(41.2)$ & $102(56.4)$ & $145(62.8)$ & $<0.001$ \\
\hline \multicolumn{6}{|l|}{ D-dimers, mg/l } \\
\hline $\begin{array}{l}<200 \\
200-400 \\
>400 \\
\end{array}$ & $205(333.04 \pm 274.44)$ & $\begin{array}{c}78(41.7) \\
92(49.2) \\
17(9.1) \\
\end{array}$ & $\begin{array}{l}57(31.5) \\
89(49.2) \\
35(19.3) \\
\end{array}$ & $\begin{array}{c}41(17.7) \\
115(49.8) \\
75(32.5) \\
\end{array}$ & $<0.001$ \\
\hline \multicolumn{6}{|l|}{ C-reactive protein, $\mathrm{mg} / \mathrm{l}$} \\
\hline $\begin{array}{l}<50 \\
50-100 \\
>100 \\
\end{array}$ & $89(110.07 \pm 90.85)$ & $\begin{array}{c}105(56.1) \\
55(29.4) \\
27(14.4) \\
\end{array}$ & $\begin{array}{l}51(28.2) \\
52(28.7) \\
78(43.1) \\
\end{array}$ & $\begin{array}{c}27(11.7) \\
53(22.9) \\
151(65.4) \\
\end{array}$ & $<0.001$ \\
\hline \multicolumn{6}{|l|}{ IL-6, pg/ml } \\
\hline $\begin{array}{l}<16(\mathrm{n}=49) \\
\geq 16(\mathrm{n}=111) \\
\end{array}$ & $45.67(169.88 \pm 534.261)$ & $\begin{array}{l}5(65) \\
8(35) \\
\end{array}$ & $\begin{array}{l}22(46.8) \\
25(53.2) \\
\end{array}$ & $\begin{array}{l}12(13) \\
78(87)\end{array}$ & $<0.001$ \\
\hline \multicolumn{6}{|l|}{ Serum Ferritin, ug/l } \\
\hline $\begin{array}{l}<300 \\
300-1000 \\
>1000 \\
\end{array}$ & $800(1231 \pm 1636.28)$ & $\begin{array}{l}70(37.4) \\
77(41.2) \\
40(21.4) \\
\end{array}$ & $\begin{array}{l}21(11.6) \\
72(39.8) \\
88(48.6) \\
\end{array}$ & $\begin{array}{c}22(9.5) \\
86(37.2) \\
123(53.2) \\
\end{array}$ & $<0.001$ \\
\hline \multicolumn{6}{|l|}{ LDH, IU/I } \\
\hline $\begin{array}{l}<280 \\
\geq 280 \\
\end{array}$ & $478(596.41 \pm 399.28)$ & $\begin{array}{c}75(40.1) \\
112(59.9) \\
\end{array}$ & $\begin{array}{c}11(6.1) \\
170(93.9) \\
\end{array}$ & $\begin{array}{c}14(6.1) \\
217(93.9) \\
\end{array}$ & $<0.001$ \\
\hline \multicolumn{6}{|l|}{ Blood group n (\%) } \\
\hline $\begin{array}{l}\text { AYE }(n=68) \\
\text { BEE }(n=79) \\
\text { AB }(n=18) \\
\text { O }(n=68)\end{array}$ & & $\begin{array}{c}13(19.1) \\
15(18.9) \\
4(22.2) \\
14(20.5) \\
\end{array}$ & $\begin{array}{c}14(20.5) \\
25(31.6) \\
9(50) \\
22(32.3) \\
\end{array}$ & $\begin{array}{c}41(60.4) \\
39(50.5) \\
5(27.8) \\
32(47.2) \\
\end{array}$ & $<0.001$ \\
\hline
\end{tabular}

$<0.001)$. Mortality rates followed the same suit with highest being in patients more than 65 years 50 (25.6\%) followed by $36(12 \%)$ in $45-65$ years and lowest 6 $(5.7 \%)$ in patients younger than $45(p<0.001)$. Deaths in the male population were $71(14.5 \%)$ compared to 21 days). An overall survival (OS) and disease severity associated survival are shown in fig-1.

Figure Legend: Moderate $(\mathrm{n}=188)$ blue line, OS $97 \%$; severe $(n=182)$ green line, OS $92.3 \%$; critical $(n=$ 
230 brown line, Cum; cumulative, OS $84.7 \%$ : log rank $<0.001$.

Available laboratory parameters of 549 patients are provided in table-II. Patients with severe and critical disease had low ALC and platelets $(p<0.001$ for both) while higher white blood cell count (WBC), absolute neutrophil count (ANC), neutrophil lymphocyte ratio (NLR), d-dimers, CRP, IL-6 and LDH levels (all $p<0.001$ ). Patients with blood group AYE had more critical disease compared to other blood groups $(p=0.03)$.

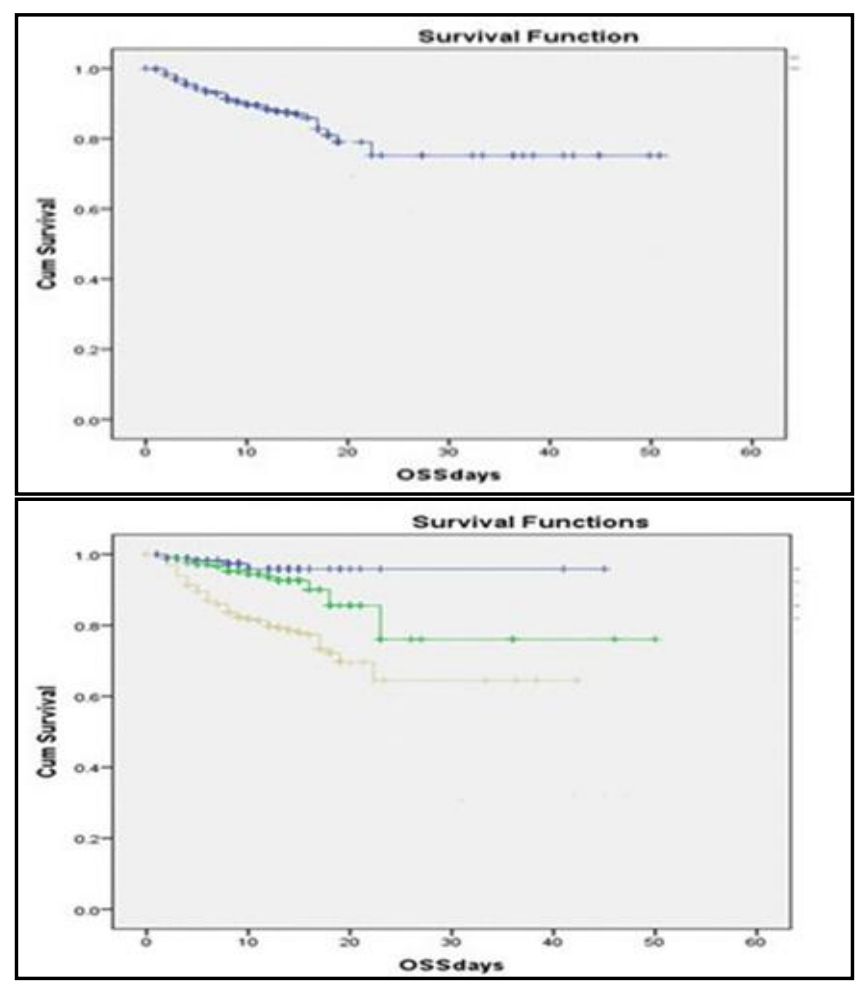

Figure: Overall survival and survival according to disease severity. A): Overall survival (OS) of the study cohort. B): OS according to disease severity.

Presence of comorbidity was a significant risk factor for mortality as $83(90.2 \%)$ deaths occurred in patients with at least one comorbidity, $(p<0.001)$, however no significant association of comorbidities was found with complications (complications $=75.3 \%$ with comorbid vs $67.5 \%$ without comorbid); $(p=0.063)$ except DM, which was significantly associated with mortality and the development of complications. Out of 228 patients with DM, $42(18.4 \%)$ died and $188(82.5 \%)$ developed complications, $(p<0.001)$. Out of $187(31.2 \%)$ patients with HTN, 31 (16.6\%) died and 141 (75.4\%) developed complications, which was not significantly different from the overall mortality and complication rate $(p=0.441)$. Similarly, other comorbidities such as
CAD ( $n=115$, Obstructive air way disease $(n=33)$, chronic kidney disease $(n=25)$, chronic liver disease $(n=6)$ and Malignancies $(n=11)$ had no significant correlation with mortality and complications ( $p>0.05$ for each).

\section{DISCUSSION}

In this study of moderate, severe and critical patients with laboratory confirmed COVID-19, the majority were men and with comorbidities and majority ended up with various complications and/or required any form of respiratory support. CRS was the commonest complication. Gender analysis by Bwire et al ${ }^{10}$ over affected Chinese population and large American study suggests that male gender is a risk factor for contracting COVID-19. Our study also showed majority of affected population being male. In our study, many findings such as association of disease severity with increasing age, symptomology of the disease and the prevalence of different comorbidities such as DM, HTN and CAD either alone or in combination are echoed with recently published studies across the globe $^{2,11}$. DM in our study was significantly associated with mortality, severity and complications, which correlates with the result of a metanalysis of 33 studies $^{12}$. In comparison to $10 \%$ prevalence of DM in COVID-19 in this metanalysis, our study showed a much higher prevalence likely due to higher overall prevalence of DM $(26.3 \%)$ in Pakistan ${ }^{13}$. The typical pattern on HRCT of COVID-19 was common in our study and it correlates closely with findings of a review article ${ }^{14}$. Lymphopenia is most frequently encountered abnormality in $35-83 \%$ patients and is reported to be associated with the severity of disease ${ }^{15,16}$ and similar results are found in our research. In our study, lymphopenia was present in $300(52.4 \%)$ patients, being highest in critical disease $(69.5 \%)$. Similar to studies from Wuhan ${ }^{17}$, Thrombocytopenia was present in $143(26 \%)$ patients in our study, $17.1 \%$ in moderate and $34.3 \%$ in critically ill patient and similar results were found in a metanalysis of 9 studies $^{18}$. Higher CRP, Ferritin, D-dimers and IL-6 in our study correlated with development of CRS and ARDS as seen in a study on risk factors of ARDS done in Wuhan ${ }^{19}$. This study could find an association of blood group Aye with critical disease but not enough evidence in favor of this finding is available in published literature. NIV was required in $29.8 \%$ in our study, which is similar to the relatively larger retrospective study at China where $32 \%$ patients required NIV ${ }^{16}$. Overall mortality in our study was $15.3 \%$, which is similar to Huang et al study done in China during the earliest days of this pandemic. However, this study 
had a small sample size $(n=41)$. A study with $72-314$ cases reported by the Chinese Center for Disease Control and Prevention showed that the case-fatality rate was $49 \%$ among critical cases compared to $31.3 \%$ in our study ${ }^{20}$. The lower mortality in our study might be because of the relatively younger population compared to west ${ }^{5}$ and use of steroids and anticoagulants in all irrespective of disease severity. Mortality rates in non-ventilated (4.9\%), NIV (21.2\%) and IV (66.6\%) patients in our study are lower to that of $6.4 \%, 40.8 \%$, and $92 \%$, respectively, compared to study done at Wuhan over 469 patients ${ }^{21}$.

CRS was the commonest complication (57.5\%) complication in our research. A strong association of COVID-19 triggered CRS with respiratory failure and ARDS has also been described previously ${ }^{22}$ but no study has calculated the incidence of CRS in COVID-19 so far. Initial studies from hospitals in Wuhan city in Mainland China ${ }^{23}$ had reported an incidence of ARDS $17-29 \%$. In our study $10.3 \%$ patients developed uncontrolled hyperglycemia. It has been noted that patients hospitalized with COVID-19 have exhibited a range of abnormalities of glucose metabolism, including worsened hyperglycemia and hyper-glycemia in patients without diabetes ${ }^{24}$. Acute liver injury (ALI) occurred in $14 \%$ patients in our study, comparable to findings in a recent systematic review incorporating 12 studies showing pooled prevalence of liver function abnormalities at $19 \%$ with an association with disease severity ${ }^{25}$. Association of positive PCR with mortality is not found in published data. The power of this research is to encompass the whole disease spectrum including complications. Incidence of CRS and post COVID fibrosis were not previously documented but it is calculated in our research.

\section{LIMITATION OF STUDY}

This study had few limitations. First, this was a retrospective study. Second, we could not follow patients longer after discharge although the course of disease is longer. Especially, prevalence of Post COVID lung and mortality could significantly alter if longer follow-up was possible.

\section{ACKNOWLEDGMENT}

We want to express my deep gratitude to Professor Dr. Shazia Nisar, for her patient guidance, enthusiastic encouragement and useful critiques of this research work. My grateful thanks are also extended to my colleagues; Dr. Rizwan Azam, Dr. Maryam Hussain, Dr. Kumail Abbass Khan and Dr Yousaf Jamal for their help in collecting the data.

\section{CONCLUSION}

In this study of moderate, severe and critically ill patients with COVID-19, we demonstrated that most Pakistani affected were men with comorbidities. A clear majority of patients developed complications and /or required respiratory support. Mortality was associated with increasing age, presence of comorbidities, especially DM and invasive ventilation.

\section{CONFLICT OF INTEREST}

This study has no conflict of interest to be declared by any author.

\section{REFERENCES}

1. WHO coronavirus disease (COVID-19) dashboard. Geneva: World Health Organization, 2020. Available online: https:// covid19.who.int/.

2. Richardson S, Hirsch J, Narasimhan M, Crawford J, McGinn T, Davidson K, et al. Presenting characteristics, comorbidities, and outcomes among 5700 patients hospitalized with covid-19 in the new york city area. J Am Med Assoc 2020; 323(20): 2052.

3. Wu Z, McGoogan J. Characteristics of and important lessons from the coronavirus disease 2019 (COVID-19) Outbreak in China. J Am Med Assoc 2020; 323(13): 1239.

4. Nishtar S, Bile K, Ahmed A, Amjad S, Iqbal A. Integrated Population-Based Surveillance of Noncommunicable Diseases. Am J Prev Med 2005; 29(5): 102-106.

5. World Bank. World Bank staff estimates based on age/sex distributions of United Nations Population Division's World Population Prospects: 2019 Revision. Available from: https:// data.worldbank.org/indicator/SP.POP.1564.TO.ZS

6. World Health Organization. Clinical management of severe acute respiratory infection (SARI) when COVID-19 disease is suspected: interim guidance, 13 March 2020. 1st ed. WHO; 2020. Available from: https://apps.who.int/iris/handle/10665/ 331446

7. Carotti M, Salaffi F, Sarzi-Puttini P, Agostini A, Borgheresi A, Minorati D, et al. Chest CT features of coronavirus disease 2019 (COVID-19) pneumonia: key points for radiologists. Radiol Med 2020; 125(7): 636-46.

8. Clinical Management Guidelines for COVID-19 Infections, Version 2 [Internet]. Nhsrc.gov.pk. 2020. Available from: http:// www.nhsrc.gov.pk/SiteImage/Misc/files/ClinicalManagement-nfection\%20v2.pdf

9. Angus D. The acute respiratory distress syndrome. J Am Med Assoc 2012; 307(23): 1-5.

10. Bwire G. Coronavirus: Why Men are More Vulnerable to Covid19 Than Women?. SN Compr Clin Med 2020; 2(7): 874-76.

11. Guan W, Liang W, Zhao Y, Liang H, Chen Z, Li Y, et al. Comorbidity and its impact on 1590 patients with COVID-19 in China: a nationwide analysis. Eur Respir J 2020; 55(5): 2000547.

12. Kumar A, Arora A, Sharma P, Anikhindi S, Bansal N, Singla V, et al. Is diabetes mellitus associated with mortality and severity of COVID-19? A meta-analysis. Diabetes Metab Syndr 2020; 14(4): 535-45.

13. Basit A, Fawwad A, Qureshi H, Shera A. Prevalence of diabetes, pre-diabetes and associated risk factors: second National Diabetes Survey of Pakistan (NDSP), 2016-2017. Br Med J Open 2018; 8(8): e020961.

14. Sahu K, Lal A, Mishra A. An update on CT chest findings in coronavirus disease-19 (COVID-19). Heart Lung 2020; 49(5): 442-43. 
15. Fan B. Hematologic parameters in patients with COVID-19 infection: a reply. Am Jn Hematol 2020; 95(8): e215.

16. Guan W, Ni Z, Hu Y, Liang W, Ou C, He J, et al. Clinical characteristics of coronavirus disease 2019 in China. New Eng J Med 2020; 382(18): 1708-20.

17. Qin C, Zhou L, Hu Z, Zhang S, Yang S, Tao Y, et al. Dysregulation of immune response in patients with COVID-19 in Wuhan, China. SSRN Electronic Journal. 2020;.

18. Lippi G, Plebani M, Henry B. Thrombocytopenia is associated with severe coronavirus disease 2019 (COVID-19) infections: A meta-analysis. Clin Chim Acta 2020; 506: 145-48.

19. Wu C, Chen X, Cai Y, Xia J, Zhou X, Xu S, et al. Risk factors associated with acute respiratory distress syndrome and death in patients with coronavirus disease 2019 pneumonia in Wuhan, China. J Am Med Assoc Int Med 2020; 180(7): 934.

20. $\mathrm{Wu} \mathrm{Z}$, McGoogan J. Characteristics of and important lessons from the coronavirus disease 2019 (COVID-19) Outbreak in
China. J Am Med Assoc 2020; 323(13): 1239.

21. Hua J, Qian C, Luo Z, Li Q, Wang F. Invasive mechanical ventilation in COVID-19 patient management: the experience with 469 patients in Wuhan. Crit Care 2020; 24(1): 348.

22. Moore J, June C. Cytokine release syndrome in severe COVID-19. Science 2020; 368(6490): 473-74.

23. Chen N, Zhou M, Dong X, Qu J, Gong F, Han Y, et al. Epidemiological and clinical characteristics of 99 cases of 2019 novel coronavirus pneumonia in Wuhan, China: a descriptive study. Lancet 2020; 395(10223): 507-13.

24. Gupta A, Madhavan M, Sehgal K, Nair N, Mahajan S, Sehrawat $\mathrm{T}$, et al. Extrapulmonary manifestations of COVID-19. Nature Med 2020; 26(7): 1017-32.

25. Mao R, Qiu Y, He J, Tan J, Li X, Liang J, et al. Manifestations and prognosis of gastrointestinal and liver involvement in patients with COVID-19: a systematic review and meta-analysis. Lancet Gastroenterol Hepatol 2020; 5(7): 667-78. 